\title{
Findings of secondary corneal amyloidosis with ultrahigh-resolution optical coherence tomography
}

This article was published in the following Dove Press journal:

Clinical Ophthalmology

14 October 2014

Number of times this article has been viewed

\author{
Kaoru Araki-Sasaki' \\ Yasuhiro Osakabe ${ }^{2}$ \\ Hideki Fukuoka ${ }^{3}$ \\ Ryuichi Ideta ${ }^{4}$ \\ Koji Hirano ${ }^{5}$
}

'Department of Ophthalmology, Japan Community Health Care Organization, Hoshigaoka Medical Center, Hirakata,

${ }^{2}$ Department of Molecular Pathology, Tokyo Medical University, Tokyo,

${ }^{3}$ Department of Ophthalmology, Kyoto Prefectural University of Medicine, Kyoto, ${ }^{4}$ Ideta Eye Hospital, Kumamoto, ${ }^{5}$ Department of Ophthalmology, Ban Buntane Hotokukai Hospital, School of Medicine, Fujita Health University, Nagoya, Japan
Correspondence: Kaoru Araki-Sasaki Department of Ophthalmology, Japan Community Health Care Organization, Hoshigaoka Medical Center,

4-8-I Hoshigaoka, Hirakata, Osaka

573-85II, Japan

Tel +8 I 72840264 I

Fax +8I 728402266

Email sasakis@sa2.so-net.ne.jp
Purpose: To describe observations by ultrahigh-resolution optical coherence tomography (OCT) in a secondary corneal amyloidosis (SCA) patient with histological analysis of excised tissue. A unique finding under OCT of her fellow eye is also described.

Case: A 39-year-old female had suffered from trichiasis in both of her eyes for more than 30 years. Slit-lamp examination showed a milky-white soft mass on her left cornea and a linear opacity on the fellow cornea at the cilia-attached region. OCT demonstrated the presence of a mass region within a thin epithelial layer and no destruction of Bowman's layer in her left cornea. In the fellow cornea, which exhibited a linear opacity, a high-density spot in Bowman's layer was observed at the cilia-attached region covered by the epithelial layer, with normal thickness. Histological examination of the excised cornea showed that the mass was positive with both Congo red and antilactoferrin antibody.

Conclusion: SCA, amyloid gradually accumulates above Bowman's layer, occupying the epithelial layer, with no destruction of Bowman's layer until the advanced stage. A high-density spot in Bowman's layer might be the first stage of SCA.

Keywords: OCT, secondary corneal amyloidosis, lactoferrin, cornea

\section{Introduction}

Secondary corneal amyloidosis (SCA) is the deposition of aggregated protein that occurs after chronic ocular inflammations or corneal disorders, eg, keratoconus, trachoma, phlyctenular keratitis, bullous keratopathy, interstitial keratitis, syphilis, trichiasis, and spheroid degeneration. ${ }^{1-9}$ We have previously classified SCA into three clinical types: gelatinous drop-like dystrophy-like appearance, lattice corneal dystrophy-like appearance, and the combined type. ${ }^{10}$ However, the details of SCA progression have yet to be clarified. Since corneal scraping or superficial keratectomy is usually only applied in advanced cases, we have not been able to obtain any samples from the very early stages that would reveal the first event of this disease.

Optical coherence tomography (OCT) is a useful tool for obtaining detailed images of the in vivo structure of the cornea. There have been many reports on using OCT to examine the morphologic structure that is present during corneal diseases. ${ }^{11,12}$ Recently, Hurmeric et al and Roszkowska et al used OCT to obtain high-quality images of the morphology of the cornea in Salzmann nodular degeneration. ${ }^{13,14}$ In our current study, we used OCT to observe the corneal structure of both eyes in SCA patient with histological analysis, especially lactoferrin expression, which is one of the precursor proteins of SCA. ${ }^{15,16}$ 


\section{Case}

A 39-year-old female who had suffered from trichiasis for more than 30 years complained of a foreign-body sensation and epiphora. The corrected visual acuity of her left eye was 20/30. Slit-lamp examination revealed multiple milky-white soft masses on the corneal surface of her left eye (Figure 1A). A slight opacity was suspected in the anterior stroma under the slit-lamp examination. In accordance with our previous classification guidelines, this mass was classified as having a gelatinous drop-like dystrophy-like appearance. ${ }^{10}$ These multiple masses were located at the cilia-attached region.

OCT (Cirrus $^{\mathrm{TM}}$ HD-OCT; Carl Zeiss, Jena, Germany; cube $4 \times 4 \mathrm{~mm}, 512 \mathrm{~A}$-scan, five-line raster $3 \mathrm{~mm}, \mathrm{~A}$-scans) revealed that while there was a large mass under the thinned epithelial layer, there was no destruction on Bowman's layer throughout the region (Figure 1, B and C), although a little high density stromal cells were observed in the anterior stromal layer.

On the other hand, the fellow cornea exhibited a linear subepithelial opacity that was not stained by fluorescein when observed under a slit-lamp examination (Figure, 2A and B). OCT revealed a high-density spot in Bowman's layer (Figure 2C), and this spot was coincident with the cilia-attached region and linear line observed under slit-lamp examination. There was normal thickness for the epithelial layer, and no change was observed in any other parts of the cornea in the fellow eye.

\section{Histological examination methods}

To resolve the foreign-body sensation in the patient, the corneal tissues were excised by lamellar keratoplasty. After these excised specimens were frozen in 30\% sucrose, $3 \mu \mathrm{m}$ sections were cut and then mounted on slides. After the slides were dried, samples were fixed with $10 \%$ formaldehyde and stained with Congo red and antilactoferrin antibody (2B8; Abcam, Cambridge, UK). All of the sections were incubated with $1 \%$ bovine serum albumin in phosphate-buffered saline at room temperature for 10 minutes each in order to block the nonspecific binding. Subsequently, the samples were then incubated with antilactoferrin antibody for 90 minutes at room temperature. The sections were washed three times in phosphate-buffered saline for 10 minutes, with the binding of the antibodies followed by reaction with biotinylated goat antirabbit immunoglobulin $\mathrm{G}$ and horseradish peroxidase-conjugated streptavidin (Histofine SAB-PO kit; Nichirei, Tokyo, Japan). The slides were dehydrated using an ethanol series (70\%-95\%) and xylene, after which they were covered with a coverslip using mounting medium. All slides were examined by both light and polarizing microscopy.

Histological analysis showed that the eosinophilic material was positively stained, with Congo red showing applegreen birefringence under polarized light (Figure 3, A-C). The material was also positive when using the antilactoferrin antibody (Figure 3D), with this area matching the Congo redpositive region. However, it should be noted that we found that Bowman's layer was occasionally destroyed within the frozen section.

Ten months after the operation, the corrected visual acuity of the patient's left eye was 20/20. Epilation of the cilia is performed regularly, and no recurrence of amyloid deposition has been found.
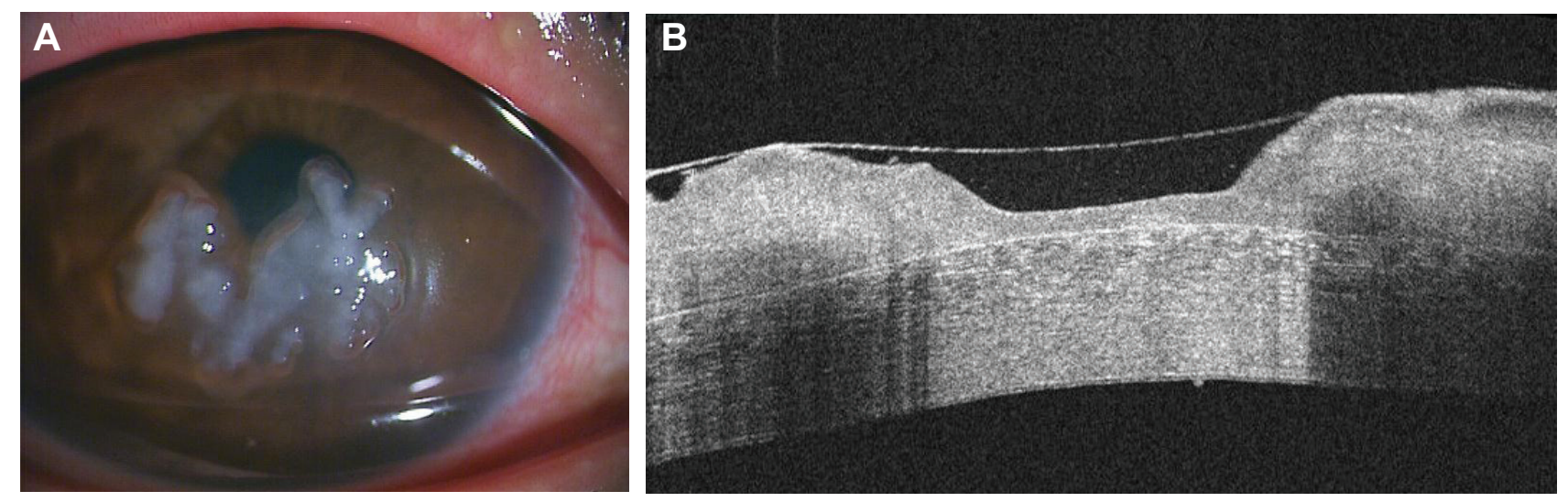

Figure I (A) Slit-lamp examination photograph of the left eye of a 39-year-old female. A white milky mass is noted in the center of the cornea with mild ciliary injection. The patient had suffered from trichiasis for more than 30 years. (B) Ultrahigh-resolution optical coherence tomography image of the left eye. The image shows homogeneous material above Bowman's layer. The material occupied the epithelial layer and caused thinning of the layer. In contrast, Bowman's layer was intact throughout the entire pathological region. 

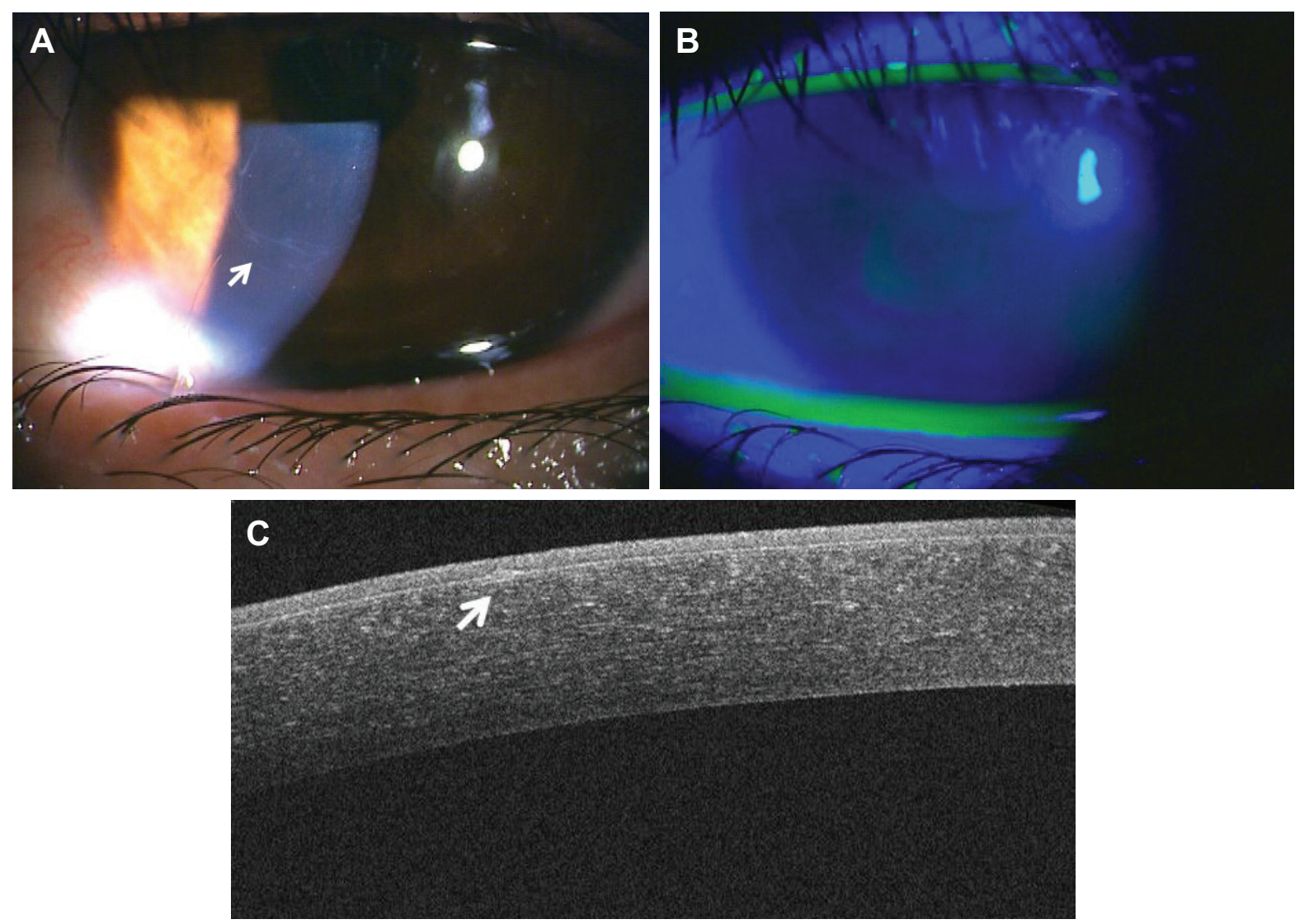

Figure 2 (A) Slit-lamp examination photograph of the right cornea. Linear opacity was observed at the cilia-attached region (arrow). (B) Slit-lamp examination shows that fluorescein did not stain the epithelial layer. (C) Optical coherence tomography indicates a high-density spot that is coincident with the linear opacity at Bowman's layer (arrow). No other remarkable changes were seen in the stroma.

\section{Discussion}

By utilizing OCT, our current study proposed two hypotheses in the pathogenicity of SCA. One is the first event of SCA. Although the high-density spot in Bowman's layer was clearly observed in the fellow eye, we could not clarify whether this was the early step of secondary corneal amyloidosis or not. This might have been a thickened Bowman's layer or an accumulation of some materials at the level of Bowman's layer. However, 1) the white mass in her left eye was confirmed as SCA by histological analysis, 2) the amyloid deposits within the epithelial layer were just above Bowman's layer in SCA, and 3) the high-density spot in Bowman's layer in the fellow eye was coincidentally located at the cilia-attached region. Therefore, we want to propose that the high-density spot in Bowman's layer in the fellow cornea might be the first step of SCA. In order to reveal this hypothesis, the linear opacity will need to be followed up for many years.

A second hypothesis is that SCA might occur within the epithelial layer, and Bowman's destruction, which is often observed in excised SCA, might be the secondary and terminal phenomenon. In our case, which was an advanced stage of SCA, we showed a uniform Bowman's layer under OCT observation.

Previous studies have reported that Bowman's layer is sometimes destroyed by amyloid aggregation. ${ }^{2,5,6,9}$ Usually, tissue sampling during the early stages is difficult to perform from an ethical point of view, and we were only able to examine the histology of advanced cases of SCA. Moreover, these histological examinations do not always reflect the in vivo structure. Artificial insults that occur during tissue preparation might also affect the histological results, as was seen in our current analysis. Our results suggest that the destruction of Bowman's layer is a secondary phenomenon, and thus will be observed only in the last stages of SCA.

Lamellar keratoplasty was chosen for the patient's left eye because of the slight changes suspected in the anterior stroma under the slit-lamp examination and OCT, considering the possibility of a functional disorder of Bowman's layer and the anterior stroma. Although we could not determine if the abnormality exists in Bowman's layer or stroma, keratectomy or epithelial scraping should be chosen as the first choice 

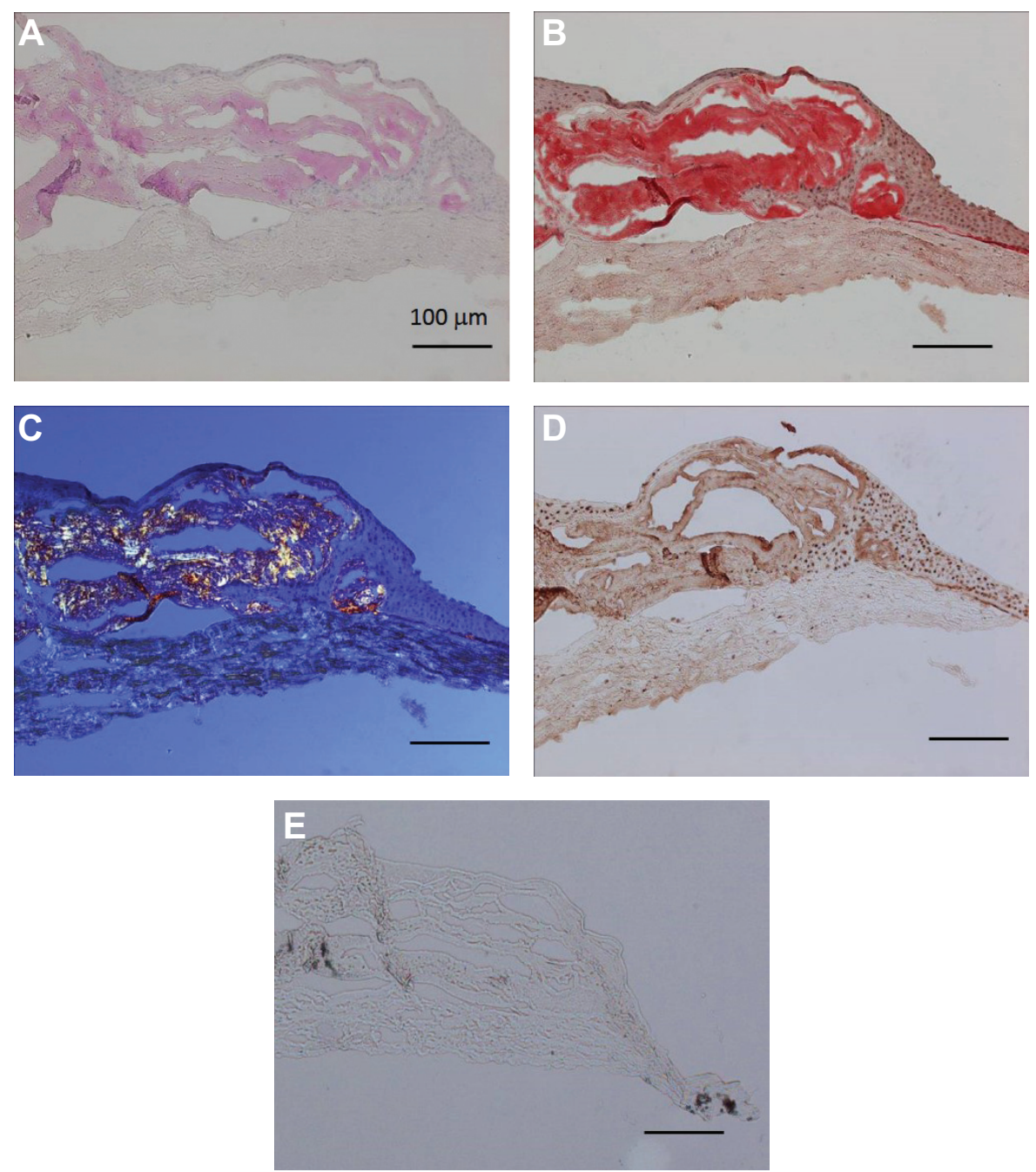

Figure 3 Immunohistochemistry of the corneal tissue excised from the left cornea.

Notes: Homogeneous material was positive with hematoxylin and eosin staining (A) and Congo red (B), and was depicted as apple-green under polarized microscopy (C). The mass region was also positively stained by the antilactoferrin antibody (D). The control that lacked the first antibody was negative (E).

for this patient. Fortunately, her visual acuity was retained after the lamellar keratoplasty, and no adverse reaction or recurrence of SCA has been found so far.

In our current study, histological analysis showed that the mass consisted of amyloid and lactoferrin, as was supported by our previous report that lactoferrin is the precursor protein of $\mathrm{SCA},{ }^{16}$ although keratoepithelin also may be associated with the pathogenesis of SCA. ${ }^{17}$ Although the main origin of lactoferrin on the ocular surface comes from tears, it is also well known that corneal epithelial cells also can secrete lactoferrin. ${ }^{18}$ Regardless of whether the origin of lactoferrin is from tears or from the corneal epithelial cells, lactoferrin does accumulate above Bowman's layer and aggregates to amyloid, creating a mass that occupies the epithelial layer without destroying Bowman's layer during the process of SCA.
Epithelial layer thinning observed under OCT has also been reported in Salzmann nodular corneal dystrophy..$^{13}$ This is a common phenomenon of diseases caused by the accumulation of various materials beneath the epithelium.

We have proposed two hypotheses on the pathogenesis of SCA. To definitively clarify our current proposals, further follow-ups of our case are necessary to reveal the details of the amyloid-accumulation process in SCA.

\section{Acknowledgment}

The authors would like to thank Dr Satoshi Kawasaki and Professor Shigeru Kinoshita for their helpful support in carrying out this study.

\section{Disclosure}

The authors report no conflicts of interest in this work. 


\section{References}

1. Stafford WR, Fine BS. Amyloidosis of the cornea. Report of a case without conjunctival involvement. Arch Ophthalmol. 1966;75:53-56.

2. Hayasaka S, Setogawa T, Ohmura M. Secondary localized amyloidosis of the cornea caused by trichiasis. Ophthalmologica. 1987;194:77-81.

3. Aldave AJ, Principe AH, Lin DY, Yellore VS, Small KW. Lattice dystrophy-like localized amyloidosis of the cornea secondary to trichiasis. Cornea. 2005;24:112-115.

4. Lin PY, Kao SC, Hsueh KF, et al. Localized amyloidosis of the cornea secondary to trichiasis: clinical course and pathogenesis. Cornea. 2003; 22:491-494.

5. Stern GA, Knapp A, Hood CI. Corneal amyloidosis associated with keratoconus. Ophthalmology. 1988;95:52-55.

6. Klemen UM, Kulnig W, Radda TM. Secondary corneal amyloidosis: clinical and pathohistological examinations. Graefes Arch Clin Exp Ophthalmol. 1983;220:130-138.

7. Rodrigues M, Zimmerman LE. Secondary amyloidosis in ocular leprosy. Arch Ophthalmol. 1971;85:277-279.

8. Santo RM, Yamaguchi T, Kanai A. Spheroidal keratopathy associated with subepithelial corneal amyloidosis. A clinicopathologic case report and a proposed new classification for spheroidal keratopathy. Ophthalmology. 1993;100:1455-1461.

9. Tai TY, Damani MR, Vo R, et al. Keratoconus associated with corneal stroma amyloid deposition containing TGFBIp. Cornea. 2009;28: 589-593.

10. Araki-Sasaki K, Hirano K, Osakabe Y, et al. Classification of secondary corneal amyloidosis and involvement of lactoferrin. Ophthalmology. 2013;120:1166-1172.
11. Correa-Pérez ME, López-Miguel A, Miranda-Anta S, Iglesias-Cortiñas D, Alió JL, Maldonado MJ. Precision of high definition spectral-domain optical coherence tomography for measuring central corneal thickness. Invest Ophthalmol Vis Sci. 2012;6:1752-1757.

12. Shousha MA, Perez VI, Wang J, et al. Use of ultra-high-resolution optical coherence tomography to detect in vivo characteristics of Descemet's membrane in Fuchs' dystrophy. Ophthalmology. 2010;117: $1220-1227$.

13. Hurmeric V, Yoo SH, Karp CL, et al. In vivo morphologic characteristics of Salzmann nodular degeneration with ultra-high-resolution optical coherence tomography. Am J Ophthalmol. 2011;151:248-256.

14. Roszkowska AM, Aragona P, Spinella R, et al. Morphologic and confocal investigation on Salzmann nodular degeneration of the cornea. Invest Ophthalmol Vis Sci. 2011;52:5910-5919.

15. Ando Y, Nakamura M, Kai H, et al. A novel localized amyloidosis associated with lactoferrin in the cornea. Lab Invest. 2002;82:757-766.

16. Araki-Sasaki K, Ando Y, Nakamura M, et al. Lactoferrin Glu561 Asp facilitates secondary amyloidosis in the cornea. Br JOphthalmol. 2005; 89:684-688.

17. Suesskind D, Auw-Haedrich C, Schorderet DF, Munier FL, Loeffler KU. Keratoepithelin in secondary corneal amyloidosis. Graefes Arch Clin Exp Ophthalmol. 2006;244:725-731.

18. Santagati MG, La Terra Mulè S, Amico C, Pistone M, Rusciano D, Enea V. Lactoferrin expression by bovine ocular surface epithelia: a primary cell culture model to study lactoferrin gene promoter activity. Ophthalmic Res. 2005;37:270-278.
Clinical Ophthalmology

\section{Publish your work in this journal}

Clinical Ophthalmology is an international, peer-reviewed journa covering all subspecialties within ophthalmology. Key topics include: Optometry; Visual science; Pharmacology and drug therapy in eye diseases; Basic Sciences; Primary and Secondary eye care; Patient Safety and Quality of Care Improvements. This journal is indexed on

Submit your manuscript here: http://www.dovepress.com/clinical-ophthalmology-journal

\section{Dovepress}

PubMed Central and CAS, and is the official journal of The Society of Clinical Ophthalmology (SCO). The manuscript management system is completely online and includes a very quick and fair peer-review system, which is all easy to use. Visit http://www.dovepress.com/ testimonials.php to read real quotes from published authors. 